\title{
Population-specific profiling of CCL3L1 copy number of the three major ethnic groups in Malaysia and the implication on HIV susceptibility
}

\begin{abstract}
CC chemokine ligand 3 like-1 (CCL3L1) encodes for CCL3L1 protein, which is a human immunodeficiency virus (HIV) suppressive chemokine and a potent ligand of HIV CCR5 coreceptor. CCL3L1 exhibits variation in the gene copy number $(\mathrm{CN})$ and could influence HIV susceptibility through gene dosage effect. The study aims to determine the distribution of CCL3L1 CN among HIV subjects of Malay, Chinese, and Indian ethnics in Malaysia and to evaluate the impact of CCL3L1 CN on susceptibility to HIV. This study involved 182 HIV patients who attended outpatient clinics of three hospitals in Malaysia and 150 non-HIV (control) subjects. Typing of CCL3L1 CN was conducted via multiplex paralogue ratio tests (PRTs), followed by validation of the CCL3L1 CN by microsatellite analyses. Both Malay and Indian HIV subjects had the $\mathrm{CN}$ mode of two, while the $\mathrm{CN}$ mode for the Chinese was four. The CCL3L1 gene CN was found to be strongly associated with ethnicity ( $p<0.001$ ) with the diverse distribution of CCL3L1 CN between the Malay (range $=0-6$ ), Chinese (range $=0-9$ ), and Indian (range $=1-4$ ) ethnic groups. CCL3L1 CN higher than and equal to the average was associated with reduced HIV susceptibility among the Malays $(\mathrm{p}<0.05)$. However, the negative results found for the Indian and Chinese need to be further analysed in a larger sample size.
\end{abstract}

Keyword: CCL3L1HIV/AIDSHIV susceptibility; Malaysia; Ethnic group; PRT 